\title{
Pathogens and antimicrobial resistance amongst stroke patients in the intensive care unit: A five years review from Benin City, Nigeria
}

\author{
Francis Odiase, ${ }^{1}$ Patrick Lofor ${ }^{2}$ \\ ${ }^{1}$ Department of Medicine; ${ }^{2}$ Department of Medical Microbiology, University of Benin/ University of Benin \\ Teaching Hospital, Benin City, Nigeria
}

\begin{abstract}
Severe stroke may necessitate intensive care unit admission, but there is a heightened risk of acquiring infection with use of ICU devices. Data regarding infection, pathogens and microbial resistance amongst stroke patients admitted into the ICU is scanty in Nigeria. This study aims to describe the infections, pathogens and antibiotics resistance pattern amongst stroke patients admitted into the ICU. It was a retrospective study. The ICU admission records of all stroke patients at the University of Benin Teaching Hospital from January 2014 to September 2019 were reviewed. The data obtained were the demographics, the types of stroke, results of microbiological studies on endotracheal aspirates, urine specimen, blood specimen, wound swab, vascular catheters, urinary catheters and the antibiogram pattern. One hundred and eight stroke patients were admitted into the ICU during the 5-year under review. The mean age was 61.8 with $51 \%$ being females and $52 \%$ having ischemic stroke. Seventy-five percent of the stroke patient
\end{abstract}

Correspondence: Francis Odiase, Department of Medicine, University of Benin/ University of Benin Teaching Hospital, Benin City, Nigeria. Tel.: 08023544232

E-mail: Francisodiase2000@hotmail.com

Key words: Antimicrobial resitance; intensive care units; stroke.

Conflict of interest: The authors have no conflict of interest to declare

Availability of data and materials: All data generated or analyzed during this study are included in this published article.

Ethics approval and consent to participate: The Ethics and Research Committee of the University of Benin Teaching Hospital Benin City, approved this study (Approval code: ADM/E22/A/VOL.VII/1476). This study conforms to the Helsinki Declaration of 1964, as revised in 2013, as it concerns human and animal rights. This was a retrospective study, reviewing medical records, hence not requiring any persons to sign consent forms.

Received for publication: 4 September 2021.

Revision received: 25 October 2021

Accepted for publication: 25 October 2021.

This work is licensed under a Creative Commons Attribution NonCommercial 4.0 License (CC BY-NC 4.0).

(C) Copyright: the Author(s),2020

Licensee PAGEPress, Italy

Annals of Clinical and Biomedical Research 2021; 2:162

doi:10.4081/acbr.2021.162 had hospital acquired infection. Ventilator associated pneumonia accounted for $67.1 \%$ of infections, urinary tract infection was $22.8 \%$, and blood stream infection $6.3 \%$ while $3.7 \%$ had infected decubitus ulcers. Microbial isolates where, Enterobacter sakazakii, accounting for $43.5 \%$, Klebsiella pneumonia 13\%, Escherichia coli $11.1 \%$, and Proteus mirabilis $7.4 \%$ while $48 \%$ had Plasmodium falciparum infection. The micro-bacteria isolates were multi-antibiotics resistant, with the highest resistance for cotrimazole, cefuroxime and ceftazidime. The stroke patient in the ICU is susceptible to developing drug resistant hospital acquired infections, which could increase mortality. Ensuring minimal cases of ICU infection with continuous antimicrobial surveillance and robust antibiotics policy should be the goal.

\section{Introduction}

Stroke is a leading cause of morbidity and mortality worldwide, with an even higher incidence in black populations. ${ }^{1,2}$ Patients suffering a severe stroke may require close monitoring and resuscitation in the intensive care unit to prevent deterioration, sustain clinical stabilization and improvement. The indications for stroke patient admission into the intensive care unit, are varied including the need for mechanical intubation for ventilation, raised ICP with poor response to interventions, unresponsive epilepticus, worsening levels of unconsciousness, general medical complications such as pulmonary embolism, cardiac arrhythmias, hypotension or overwhelming septicemia with hemodynamic instability. ${ }^{3-5}$ Additionally, extensive acute middle cerebral artery occlusion, acute basilar occlusion and cerebellar or brainstem hemorrhage could result in ICU admission. ${ }^{6,7}$ The assessment, care and resuscitation the stroke patients undergoes in the ICU involves meticulous medical, nursing and physiotherapy care, including maintenance of respiratory and cardiovascular stability

The admission of patients into the ICU is not without its challenges including, the acquisition of infection. Hospital-acquired infections are highly prevalent in the ICU and these have been associated with the use of invasive devices, including endotracheal tubes, vascular and urinary catheters. The severity of the clinical state of ICU patients, the need for frequent antibiotic use and the development of antimicrobial resistance does complicates the situation. ${ }^{8-11}$ In low and middle-income countries the rate of hospital acquired infection is about $5.7 \%$ to $19.1 \%$ according to WHO estimates, but with an even higher infection rate in the ICU ranging from $4.4 \%$ to $88.9 \% .^{12}$ It has been observed that in the developing world, the infection rate in the ICU is three times as high as in the western world. ${ }^{10,12}$

A 14-nation European study revealed that $8 \%$ of patients on admission in the ICU for more than two days had at least one ICUacquired infection, with $6 \%$ developing pneumonia, $4 \%$ having bloodstream infection and $3 \%$ with urinary tract infection. Ninety- 
eight percent of pneumonia and $48 \%$ of UTI was associated with intubation and catheterization. ${ }^{13-15}$

The most isolated micro-organism in this large study included Pseudomonas, Staphylococcus aureus, Escherichia coli, klebsiella spp, and Enterobacter Acinetobacter baumannii. ${ }^{13-15}$

The patients on admission in the ICU are already in critical, poor clinical conditions and having infections with resistant microbes can only compound their situation, which would translate to prolonged admission, additional costs, and higher mortality. ${ }^{9-11}$

To the best of our knowledge we are not aware of any study that has described the prevalence, pattern of infections, microbial isolates and antibiotics resistance amongst stroke patients admitted into ICU in Nigeria. Earlier studies in Nigeria ${ }^{16-20}$ and other parts of Africa ${ }^{21-24}$ regarding infections in the ICU involved all ICUadmitted patients irrespective of the diagnosis.

This study aims to describe the infections and pathogens the ICU-stroke admitted patients acquires and the resistance pattern of the microbial isolates. This would provide an understanding of the infection profile, guide therapy of these ICU-stroke admitted patients and strengthen infection control measures in critical care settings.

\section{Materials and Methods}

This was a retrospective study. It involved the review of the medical records of all the stroke patients admitted into the intensive care unit at the University of Benin Teaching Hospital, from January 2014 to September 2019. The ICU is a nine bedded, mixed unit, for medical and surgical cases with two specialist anesthetists and several residents. It is capable of providing complex multi-system life support. Ethical approval was obtained from the Ethics and Research Committee of the University of Benin Teaching Hospital. The review of the medical records was based on the Center for Disease Control (CDC) definition of hospital acquired infection, which is any infection acquired and developing at least 48-72 hours after admission, and the device-related infection as defined by the National Nosocomial Infection Surveillance System (NNIS). ${ }^{25,26}$

The paper records showed that microscopy, culture, speciation and sensitivity was done by one of the authors, on the following: i) Swab from materials for intubation; ii) Blood film for malaria parasite, thick blood film by oil immersion microscopy x 100 objective; ii) Culture of bronchial lavage aspirate; iv) Intravenous catheter specimen for MCS; v) Urine Catheter specimen for MCS; vi) Blood culture by BACTEC method; vii) Swab from decubitus ulcer for MCS. All specimens following collection were transported immediately to the microbiology laboratory within a space of 15 minutes. Antimicrobial susceptibility screening used eleven different antibiotics during the 5-year period under review, with six to eight antibiotics disc at a time per agar plate by the Kirby Bauer disc diffusion technique. The incubation period for Plasmodium falciparum is 8 to 14 days, therefore a new case of malaria was determined by parasitaemia occurring on day $8^{\text {th }}$ of admission with symptoms or symptoms following transfusion of blood containing malaria trophozoites. ${ }^{27}$ Infection of intravenous catheter determined when culture of tip of intravenous catheter had a growth of 15 colony forming units $/ \mathrm{mL}$ and above. Urine and urine catheter tip growth of more than $10^{5}$ colony forming unit (C.F.U) $/ \mathrm{mL}$. Any growth from the blood culture using the BACTEC method is significant. Intubation devices are meant to be sterile so pure growth from intubation materials indicate infection. Data extracted using a standard pro forma included age, gender, type of stroke, year of admission, microbial isolates and antibiogram. The statistical analysis was performed using SPSS version 21. This was a descriptive study and the main analysis was descriptive, with continuous variables presented as means, median, standard deviation and range while frequency and percentages was used to summarize categorical variables.

\section{Results}

One hundred and eight stroke patients were admitted into the intensive care unit between January 2014 and September 2019. The mean age was 61.8 with an age range of 38 to 95years. The age range 66 to 70 years had the highest numbers of stroke ICU admission. Fifty-two percent had ischemic stroke while $48 \%$ were hemorrhagic stroke. Fifty-three of them were males while 55 were females. Seventy-five percent of the stroke patient on admission in the ICU had hospital acquired infection. Fifty-two had malaria infestation, which accounted for $48 \%$ of the infections of which $39.7 \%$ are males and $60.3 \%$ were females, Table 1 . The total numbers of isolates had increased over time with the malaria isolate increasing more in the last three years. About $86.5 \%$ (45/52) of the stroke patient who had blood transfusion had malaria parasitemia. Amongst the bacteria isolates, Enterobacter sakazakii accounted for $43.5 \%$, of infections, while Klebsiella pneumonia 13\%, Escherichia coli $11.1 \%$, and Proteus mirabilis $7.4 \%$. The microbacteria isolates were multi-antibiotics resistant, with organisms having the highest resistance for co-trimazole, cefuroxime, ceftazidime. There is also some resistance to co-amoxiclav and ceftriaxone. Escherichia coli and Klebsiella pneumonia showed resistance to virtually all the earlier antibiotics before meropenem introduction Table 2. Isolates were obtained from, fifty-two blood specimen materials (malaria-parasites), fifty-three endotracheal specimen materials (Enterobacter sakazakii, Klebselle pneumonia), 18 urine samples (Enterobacter sakazaki, Escherichia coli, Proteus mirabilis, Klebselle pneumonia), 5 blood culture specimen, (Enterobacter sakazaki) and 3 decubitus ulcers specimens (Proteus mirabilis). The infection pattern comprised of ventilator associated pneumonia accounting for $67.1 \%$ of infections, urinary tract infection $22.8 \%$, and blood stream infection $6.3 \%$ while $3.7 \%$ had infected decubitus ulcers.

\section{Discussion}

This study revealed that about three-quarters $(75 \%)$ of the ICU-admitted stroke patients had Hospital acquired infections, this is the observation in several studies in which though the numbers of beds in the intensive care unit are proportionally small compared to the other wards, it is said to have about the highest rates of hospital acquired infections in many centers. $9,10,15,28,29$ The poor admitting clinical state of patients, the need for invasive devices, extended admission period and prolonged antibiotics use could be accountable. $9,10,15,16,21$

This study revealed that in the period under review (2017 to 2019), the year following had more microbial isolates than the previous year. It is possible there was a yearly increase in ICU admissions of the stroke patients especially with increased awareness of stroke and the availability of neuro-imaging that assist in diagnosis and assessment of severity of the stroke. Such an increase in the infection pattern over time have similarly been observed. ${ }^{9,10,28,29}$

A marginally more female stroke patient than male patients were admitted into the ICU in the study, this is unlike the general 
higher prevalence of stroke occurring in males than in females, most likely this picture is reflective of severity or clinical deterioration requiring attention. Those in their prime of life, the middle aged and elderly were the most stroke patients in the ICU from our study. The lower prevalence in the very old might be explained by the higher mortality in this age group, while the few numbers in younger age is in keeping with the fact that this age is the least demographic group at risk for stroke. The stroke type admitted into the ICU are about the same in number, again this is in contrast to the pattern of a higher proportion of ischemic strokes than hemorrhagic in the community or stroke ward, as the ICU is dedicated for a more sever stroke irrespective of the type.

Plasmodium Falciparium malaria was the most prevalent infection amongst the stroke patients admitted into the ICU in this study. This is explained by the fact that the ICU is located in a malaria endemic area, with patients exposed easily to infected mosquitoes. Although the windows and doors are netted, with the opening of the doors the mosquitoes gain entrance into the unit and infect patients since their individual beds are not netted. Additionally there is non-utilization of insecticides because of the clinical conditions of patients. Majority of these patients in the
ICU have poor clinical conditions requiring blood transfusion, and with regards to our stroke patients over three-quarters, had transfusion and this is a source of transmission of the malaria parasites. It is quiet noteworthy that this pattern is made obvious from this study as fever in the ICU could easily be thought to be due to some more exotic causes without consideration of the commoner malaria fever, such an omission can only cause further deterioration in the patient's condition as fever is known to impact negatively to stroke recovery. Majority of these ICU-admitted stroke patients developed resistant Plasmodium Falciparium infections, since repeated microbiological studies were positive for Plasmodium Falciparium in spite of adequate antimalarial treatments, hence the need for closer microbiological studies in these patients.

The bacteria isolates in this study were mainly gram-negative organism, which has similarly been observed in other works. ${ }^{30,31}$ Enterobacter sakazakii was the most isolated bacteria in this study, a gram negative motile rod. It is a particularly rare strain of the Enterobacteriaceae family, a ubiquitous organism found in the environment, in humans and plants and resistant to protocol meant to ensure hygiene in the hospital. ${ }^{32}$ In this study it was isolated from endotracheal materials, the urine and blood, causing pneumo-

Table 1. Demographics and infection pattern of 108 stroke patients in the ICU.

\begin{tabular}{|c|c|c|c|c|}
\hline Gender & Male n (\%) & Female n (\%) & Total & \\
\hline $\begin{array}{l}\text { Age range } \\
\quad<55 \text { years } \\
55 \text { to } 64 \text { years } \\
>64 \text { years }\end{array}$ & $\begin{array}{c}6(5.6) \\
23(21.3) \\
24(22.2)\end{array}$ & $\begin{array}{c}9(8.3) \\
21(19.4) \\
25(23.1)\end{array}$ & $\begin{array}{l}15 \\
44 \\
49\end{array}$ & $\mathrm{P}=0.71$ \\
\hline $\begin{array}{l}\text { Type of stroke } \\
\text { Ischemic stroke } \\
\text { Hemorrhagic stroke }\end{array}$ & $\begin{array}{l}26(46.4) \\
27(51.9)\end{array}$ & $\begin{array}{l}30(53.6) \\
25(48.1)\end{array}$ & $\begin{array}{l}56 \\
52\end{array}$ & \\
\hline \multicolumn{5}{|l|}{ Infection } \\
\hline $\begin{array}{l}\text { Enterobacter sakazaki alone } \\
\text { Enterobacter sakazaki with malaria } \\
\text { Eschericher coli alone } \\
\text { Eschericher coli with malaria } \\
\text { Klebsiella pneumonia alone } \\
\text { Klebsiella pneumonia with malaria } \\
\text { Proteus mirabilis alone } \\
\text { Proteus mirabilis with malaria } \\
\text { Malaria infection } \\
\text { Malaria following blood transfusion }\end{array}$ & $\begin{array}{l}12(63.6) \\
14(66.0) \\
3(42.9) \\
1(20.0) \\
3(37.5) \\
1(16.7) \\
2(33.3) \\
21(39.7) \\
21(47.3)\end{array}$ & $\begin{array}{l}7(36.4) \\
7(34.0) \\
4(57.1) \\
4(80.0) \\
5(62.5) \\
5(83.3) \\
4(66.7) \\
2(100.0) \\
31(60.3) \\
24(52.7)\end{array}$ & $\begin{array}{c}19 \\
28 \\
7 \\
5 \\
8 \\
6 \\
6 \\
2 \\
52 \\
45\end{array}$ & \\
\hline
\end{tabular}

Table 2. Antibiogram of microbial isolates from 108-stroke patients in the ICU.

\begin{tabular}{|c|c|c|c|c|}
\hline & Enterobacter sakazakii & Escherichia coli & Klebsiella pneumonia & Proteus mirabilis \\
\hline Ampicillin & $\mathrm{R}$ & $\mathrm{R}$ & $\mathrm{R}$ & SSS \\
\hline Ceftriaxone & SSS & SS & $\mathrm{R}$ & $\mathrm{R}$ \\
\hline Co-trimoxazole & $\mathrm{R}$ & $\mathrm{R}$ & $\mathrm{R}$ & $\mathrm{R}$ \\
\hline Gentamicin & SS & $\mathrm{R}$ & SSS & - \\
\hline erythromycin & SS & $\mathrm{R}$ & $\mathrm{R}$ & SS \\
\hline Co-amoxiclav & SSS & $\mathrm{R}$ & $\mathrm{R}$ & - \\
\hline Levofloxacin & SSS & - & SSS & SSS \\
\hline Cefuroxime & $\mathrm{R}$ & - & $\mathrm{R}$ & $\mathrm{R}$ \\
\hline Imipanem & - & SSS & - & SS \\
\hline Meropenem & - & SSS & - & - \\
\hline Ceftazidime & $\mathrm{R}$ & $\mathrm{R}$ & $\mathrm{R}$ & $\mathrm{R}$ \\
\hline
\end{tabular}

SSS = sensitive, $\mathrm{SS}=$ intermediate sensitivity, $\mathrm{R}=$ resistant. 
nia, urinary tract infections and septicemia. This is the observation from other workers with similar infection patterns. ${ }^{33-35}$ The notoriety of Enterobacter sakazakii is said be facilitated by the poor clinical state of the patient and prolonged antibiotics exposure. Such antibiotic use alters the microbiota, which provides a favorable milieu for colonization and infection. ${ }^{33,34}$ Enterobacter sakazakii isolate showed resistance to several commonly used antibiotics including co-trimoxazole, ampicillin, and cefuroxime while having intermediate sensitivity to gentamicin and erythromycin.

Klebsiella pneumonia was the next isolated bacteria in this study. A non-motile rod, an enteric organism of the Enterobacteriaceae family. It was cultured from endotreacheal material and urine in this study, where it caused pneumonia and UTI. Infection by Klebsiella pneumonia is enabled by the poor clinical state of patient, prolonged antibiotics exposure and use of devices. ${ }^{34-36}$ This colonization of the respiratory tract, causing pneumonia and catheter associated UTI in the critical patient by Klesbsiella pneumonia has been reported previously. ${ }^{34-36}$ Klesbsiella pneumonia isolate was found resistant to seven frequently used antibiotics, including co-trimoxazole, erythromycin, ampicillin, co-amoxiclav, ceftriaxone, cefuroxime and ceftazidime. Klesbsiella pneumonia is known to have a high tendency for attaining resistance and similar resistance patterns have been reported. ${ }^{34-36 .}$

Escherichia coli a Gram-negative bacteria, a gut resident, was the third commonest isolate in our study, and was mainly isolated from urine. It is established as the most common causative organisms in uncomplicated and complicated UTI associated with catheter insertion. ${ }^{37-39}$ Escherichia coli was shown to be multiantibiotic resistant to ampicillin, co-trimoxazole, gentamicin, erythromycin, co-amoxiclav, and ceftazidime in our study. Wide spread Escherichia coli resistance to several antibiotics is well documented. ${ }^{37-42}$ In this study Proteus mirabilis was the least isolated, a gut resident a normal flora of fecal matter. It was isolated from the urine samples and swabs from pressure ulcers. It is flagellated and swarms considerably (highly motile organism) infecting decubitus ulcer, contaminating the urethra and colonizing the urinary catheter even at some distance. Other studies have had about the same findings. ${ }^{9,10,11,43}$ Proteus mirabilis was similarly resistant to almost all the commonly used antibiotics drugs including ceftriaxone, cotrimoxazole, cefuroxime and ceftazidime is well established. $8,9,10,31$

Majority of the isolates were sensitive to levofloxacin, while co-trimoxazole, ampicillin, cefuroxime and ceftazidime had the highest resistance. Erythromycin and ceftriaxone had about fifty percent resistant from the isolated organism. Antibiotics pressure from prolonged use and the transference of mobile genetic elements, does facilitate acquisition of resistance to commonly used antibiotics. This is in addition to natural resistance of many of these microbial isolates from the ICU. This pattern of antibiotics drug resistance narrows the choice of antibiotics and severely limits the treatment options of these stroke patients.

Therapeutic and diagnostic invasive devices are the main nidus of infection in the ICU. These involves mechanical ventilation, central venous catheterization, invasive pressure monitoring, urinary catheterization

The endotracheal materials were the commonest sources of isolate in this study, which is akin to ventilator use, with threequarter of the micro-organisms isolated from this device. Ventilator associated pneumonia is prominent in the ICU amongst device infections and has been well documented. $8,9,10,44,45$ Catheter associated UTI was the second commonest device associated infection in this study. Majority of ICU admitted patients would need catheterization for reason of quantifying the urinary output, but the stroke patient is especially likely to be catheterized. Language deficit, loss of consciousness, and neurogenic bladder are amongst the reason for this. UTI from catheter placement is a frequent occurrence in stroke patients. ${ }^{3,6,8,10}$

Blood stream infections from aerobic blood culture studies associated with use of intravenous devices was the next in this study. This pattern of ventilator associated infection then catheter associated infection followed by blood stream infections have been similarly reported. $10,31,46-49$

The numbers of isolates from pressure ulcer was least, probably because of a short transition time at the ICU, a limited time for ulcer development, frequent turning of patients and the use of air mattresses.

The study was limited by its retrospective design, with the authors having no total control over the standard or comprehensiveness of the data retrieved. The participants were not recruited prospectively, or immediately on admission into the ICU as a consequence some of the infections reported might have been acquired outside or before the ICU admission, either in the community or the stroke ward. This may have accounted for the high infection rate observed. A second limitation was that the record showed that ESBL (Extended spectrum $\beta$ lactamase) was not tested for in these gram negative isolates. Thirdly anaerobic studies were not done

\section{Conclusions}

The intensive care unit is a specialized unit with multi-disciplinary engagement, involving the use of gadgets, devices for respiratory, cardiac, and renal support, but in spite of these, acquisition of drug resistant nosocomial infections is a common occurrence and this worsens morbidity and the mortality. Ensuring minimal cases of nosocomial infections in the ICU entails multi-disciplinary approach, with continuous antimicrobial surveillance, robust antibiotics policy and stewardship. Aggressive infection control measures are needed to reduce infections in critical care settings including, preventing patient to patient spread, hand washing and equipment hygiene while avoiding unnecessary prolonged device application, training and re-training of ICU staff.

A prospective study, involving anaerobic culture studies, testing of ESBL, while using modern molecular techniques is recommended so as to give a clearer picture.

\section{References}

1. Kim J, Thayabaranathan T, Donnan GA, et al. Global Stroke Statistics 2019. Int J Stroke. 2020;15:819-38.

2. Gardener H, Sacco RL, Rundek T, et al. Race and ethnic disparities in stroke incidence in the Northern Manhattan study. Stroke 2020;51:1064-9.

3. Alonso A, Ebert AD, Kern R, et al. Outcome predictors of acute stroke patients in need of intensive care treatment. Cerebrovasc Dis 2015;40:10-7.

4. Crawshaw AA, Cock HR. Medical management of status epilepticus: Emergency room to intensive care unit. Seizure 2020;75:145-52.

5. Pinto VL, Tadi P, Adeyinka A. Increased intracranial pressure. [Updated 2021 May 4]. In: StatPearls [Internet]. Treasure Island (FL): StatPearls Publishing; 2021 Jan-.

6. de Montmollin E, Terzi N, Dupuis C, et al. One-year survival 
in acute stroke patients requiring mechanical ventilation: a multicenter cohort study. Ann Intensive Care 2020;10:53.

7. Sulter G, Elting JW, Langedijk M, et al. Admitting acute schemic stroke patients to a stroke care monitoring unit versus a conventional stroke unit: a randomized pilot study. Stroke 2003;34:101-4.

8. Despotovic A, Milosevic B, Milosevic I, et al. Hospital acquired infections in the adult intensive care unit, epidemiology, antimicrobial resistance patterns, and risk factors for acquisition and mortality. Am J Infect Control 2020;48:12115.

9. Allegranzi B, Nejad SB, Combescure C, et al. Burden of endemic health-care-associated infection in developing countries: systematic review and meta-analysis. Lancet 2011;377:228-41.

10. Rosenthall VD, Maki DG, Salomao R, et al. Device associated nosocomial infections in 55 intensive care units of 8 developing countries. Ann Intern Med 2006;145:582-91.

11. Arefian H, Hagel S, Fischer D, et al. Estimating extra length of stay due to healthcare-associated infections before and after implementation of a hospital-wide infection control program. PloS One 2019;14:e0217159.

12. World Health Organization. Health care-associated infections fact sheet. WHO; 2020. Available at: http://www.who.int/gpsc/country_work/gpsc_ccisc_fact_sheet en.pdf

13. European Centre for Disease Prevention and Control. Annual epidemiological report 2014. Antimicrobial resistance and healthcare-associated infections. Stockholm: ECDC; 2015. Available from: https://www.ecdc.europa.eu/en/publicationsdata/antimicrobial-resistance-and-healthcare-associated-infections-annual

14. European Centre for Disease Prevention and Control. Point prevalence survey of healthcare-associated infections and antimicrobial use in European acute care hospitals. Stockholm: ECDC; 2013. Available from: https://www.ecdc.europa.eu/en/publications-data/point-prevalence-survey-healthcare-associated-infections-and-antimicrobial-use-0

15. European Centre for Disease Prevention and Control. European Surveillance of Healthcare Associated Infections in Intensive Care Units-HAI-Net ICU Protocol, Version 1.02; ECDC: Stockholm, Sweden, 2015. Available online: https://www.ecdc.europa.eu/en/publications-data/europeansurveillance-healthcare-associated-infections-intensive-careunits-hai

16. Iwuafor AA, Ogunsola FT, Oladele RO, et al. Incidence, clinical outcome and risk factors of intensive care unit infections in the Lagos University Teaching Hospital (LUTH), Lagos, Nigeria. PLoS One 2016;11:e0165242.

17. BO Bolaji, IK Kolawole. The intensive care unit of the University Teaching Hospital, Ilorin, Nigeria: A ten year review (1991-2001). Southern Afr J Anaesth Analges 2005; 11:146150.

18. Yunusa T, Adeoye AM, Akitoye OA. Profile of septic work up among patients admitted into the intensive care unit in University of Abuja teaching hospital Gwagwalada, Abuja. Afr J Clin Experiment Microbiol 2019:20:9-16.

19. Iregbu K, Sonibare SA. Profile of infections in Intensive Care Unit (ICU) in a Central Nigeria tertiary hospital. Afr J Clini Exper Microbiol 2015;16:23-7.

20. Iliyasu G, Daiyab FM, Tiamiyu AB, et al. Nosocomial infections and resistance pattern of common bacterial isolates in an intensive care unit of a tertiary hospital in Nigeria: A 4-year review. J Critical Care 2016:34:116-20.

21. Agaba P, Tumukunde J, Tindimwebwa JVB, Kwizera A. Nosocomial bacterial infections and their antimicrobial susceptibility patterns among patients in Ugandan intensive care units: a cross sectional study. BMC Res Notes 2017;10:349.

22. Claver HP, Ernest A, Philippe A, et al. Epidemiology and prognostic factors of stroke in intensive care unit at the National University Hospital Hubert Koutoukou Maga (CNHU-HKM) of Cotonou. Anesthesia Critical Care 2020;2:1-7.

23. Fahim NAE. Prevalence and antimicrobial susceptibility profile of multidrug-resistant bacteria among intensive care units patients at Ain Shams University Hospitals in Egypt-a retrospective study. J Egypt Public Health Assoc 2021;96:7.

24. Bhagwanjee S, Scribante J, Paruk F. Prevalence of ICU infection in South Africa and accuracy of treating physician diagnosis and treatment. Crit Care 2009;13:P347.

25. Garner JS, Jarvis WR, Emori TG, et al. CDC definitions for nosocomial infections. Am J Infect Control 1988;16:128-40.

26. Richards MJ, Edwards JR, Culver DH, Gaynes RP. Nosocomial infections in medical intensive care units in the United States. National Nosocomial Infections Surveillance System. Crit Care Med 1999;27:887-92.

27. Long CA, Hoffman SL. Parasitology. Malaria- from infant to genomics to vaccines. Science 2002;297:345-7.

28. Vincent JL, Sakr Y, Singer M, et al. Prevalence and outcomes of infection among patients in intensive care units in 2017. JAMA 2020;323:1478-87.

29. Manchanda V, Suman U, Singh N. Implementing infection prevention and control programs when resources are limited. Curr Treat Options Infect Dis 2018;10:28-39.

30. Nazir A, Kadri S. An overview of hospital acquired infections and the role of the microbiology laboratory. Int J Res Med Sci 2017;2:21-27.

31. Yatin-Mehta Y, Jaggi N, Rosenthal VD, et al. Device-associated infection rates in 20 cities of India, data summary for 2004 2013: findings of the International Nosocomial Infection Control Consortium. Infect Control Hosp Epidemiol 2016;37:172-81.

32. Uc-Cachón AH, Gracida-Osorno C, Luna-Chi IG, et al. High prevalence of antimicrobial resistance among gram-negative isolated bacilli in intensive care units at a tertiary-care hospital in Yucatán Mexico. Medicina (Kaunas) 2019;55:588.

33. Breeuwer P, Lardeau A, Peterz M, Joosten HM. Desiccation and heat tolerance of Enterobacter sakazakii. J Appl Microbiol 2003;95:967-73.

34. Zilahi G, Artigas A, Martin-Loeches I. What's new in multidrug-resistant pathogens in the ICU? Ann Intensive Care 2016;6:96.

35. Segagni Lusignani L, Presterl E, Zatorska B, et al. Infection control and risk factors for acquisition of carbapenemase-producing enterobacteriaceae. A 5 year (2011-2016) case-control study. Antimicrob Resist Infect Control 2020;9:18.

36. Zhang H, Guo Z, Chai Y, et al Z. Risk factors for and clinical outcomes of carbapenem-resistant Klebsiella pneumoniae nosocomial infections: A retrospective study in a tertiary hospital in Beijing, China. Infect Drug Resist 2021;14:1393-1401.

37. Abduzaimovic A, Aljicevic M, Rebic V, et al. Antibiotic resistance in urinary isolates of Escherichia coli. Mater Sociomed 2016;28:416-9.

38. Ali I, Rafaque Z, Ahmed S, et al. Prevalence of multi-drug resistant uropathogenic Escherichia coli in Potohar region of Pakistan. Asian Pac J Trop Biomed 2016;6:60-6. 
39. Asadi Karam MR, Habibi M, Bouzari S. Urinary tract infection: Pathogenicity, antibiotic resistance and development of effective vaccines against Uropathogenic Escherichia coli. Mol Immunol 2019;108:56-67.

40. Hitzenbichler F, Simon M, Holzmann T, et al. Antibiotic resistance in E. coli isolates from patients with urinary tract infections presenting to the emergency department. Infection 2018;46:325-31.

41. Idil N, Candan ED, Rad AY, Aksoz N. High trimethoprim-sulfamethoxazole resistance in ciprofloxacin-resistant Escherichia coli strains isolated from urinary tract infection. Minerva Biotecnol 2016;28:159-63.

42. Prasada S, Bhat A, Bhat S, et al. Changing antibiotic susceptibility pattern in uropathogenic Escherichia coli over a period of 5 years in a tertiary care center. Infect Drug Resist 2019;12:1439-43.

43. Armbruster CE, Mobley HLT, Pearson MM. Pathogenesis of Proteus mirabilis Infection. EcoSal Plus 2018;8:0009.

44. Papazian L, Klompas M, Luyt CE. Ventilator-associated pneumonia in adults: a narrative review. Intensive Care Med
2020;46:888-906.

45. Kharel S, Bist A, Mishra SK. Ventilator-associated pneumonia among ICU patients in WHO Southeast Asian region: A systematic review. PLoS One 2021;16:e247832.

46. Gahlot R, Nigam C, Kumar V, et al. Catheter-related bloodstream infections. Int J Crit Illn Inj Sci 2014;4:162-7.

47. Braga IA, Campos PA, Gontijo-Filho PP, Ribas RM. Multihospital point prevalence study of healthcare-associated infections in 28 adult intensive care units in Brazil. J Hosp Infect 2018;99:318-24.

48. Sabino SS, Lima CA, Machado LG, et al. Infections and antimicrobial resistance in an adult intensive care unit in a Brazilian hospital and the influence of drug resistance on the thirty-day mortality among patients with bloodstream infections. Rev Soc Bras Med Trop 2020:22:e20190106.

49. Tian L, Zhang Z, Sun Z. Antimicrobial resistance trends in bloodstream infections at a large teaching hospital in China: a 20-year surveillance study (1998-2017). Antimicrob Resist Infect Control 2019:28:86. 